\title{
Sleep Slow-Wave Activity Reveals Developmental Changes in Experience-Dependent Plasticity
}

\author{
Ines Wilhelm, ${ }^{1}$ Salomé Kurth, ${ }^{1,3}$ Maya Ringli, ${ }^{1}$ Anne-Laure Mouthon, ${ }^{1}$ Andreas Buchmann, ${ }^{1,4}$ Anja Geiger, ${ }^{1}$ \\ Oskar G. Jenni, ${ }^{1,2}$ and Reto Huber ${ }^{1,2,5}$ \\ ${ }^{1}$ Child Development Center and ${ }^{2}$ Children's Research Center, University Children's Hospital Zürich, 8032 Zürich, Switzerland, ${ }^{3}$ University of Colorado at \\ Boulder, Boulder, Colorado, 80309, ${ }^{4}$ Department of Neurology, University Hospital Zürich, 8091 Zürich, Switzerland, and ${ }^{5}$ University Clinics for Child and \\ Adolescent Psychiatry, 8032 Zürich, Switzerland
}

Experience-dependent plasticity, the ability of the brain to constantly adapt to an ever-changing environment, has been suggested to be highest during childhood and to decline thereafter. However, empirical evidence for this is rather scarce. Slow-wave activity (SWA; EEG activity of 1-4.5 Hz) during deep sleep can be used as a marker of experience-dependent plasticity. For example, performing a visuomotor adaptation task in adults increased SWA during subsequent sleep over a locally restricted region of the right parietal cortex, which is known to be involved in visuomotor adaptation. Here, we investigated whether local experience-dependent changes in SWA vary as a function of brain maturation. Three age groups (children, adolescents, and adults) participated in a high-density EEG study with two conditions (baseline and adaptation) of a visuomotor learning task. Compared with the baseline condition, sleep SWA was increased after visuomotor adaptation in a cluster of eight electrodes over the right parietal cortex. The local boost in SWA was highest in children. Baseline SWA in the parietal cluster and right parietal gray matter volume, which both indicate region-specific maturation, were significantly correlated with the local increase in SWA. Our findings indicate that processes of brain maturation favor experience-dependent plasticity and determine how sensitive a specific brain region is for learning experiences. Moreover, our data confirm that SWA is a highly sensitive tool to map maturational differences in experience-dependent plasticity.

Key words: development; experience-dependent plasticity; learning; maturation; sleep; slow wave sleep

\section{Introduction}

Children spend much of their waking hours in school so as to acquire fundamental skills and knowledge about the world. This seems to make perfect sense, because the developing brain is assumed to be very sensitive to novel experiences, resulting in a high capability for learning and memory. However, little empirical evidence is actually available in humans to indicate that experiences have more profound and more long-lasting effects in early than in later life. Studies focusing on sensitive periods may give first hints on the modulating effect of age on experiencedependent plasticity. A sensitive period is a time window during development in which specific experiences have most profound effects on the brain and behavior (Knudsen, 2004). First evidence for the existence of sensitive periods in humans comes from studies on language development and musical training. In these ex-

\footnotetext{
Received March 11, 2014; revised Aug. 1, 2014; accepted Aug. 7, 2014.

Author contributions: 0.G.J. and R.H. designed research; I.W., S.K., M.R., A.-L.M., and A.G. performed research; I.W., S.K., M.R., A.-L.M., A.B., and R.H. analyzed data; I.W. and R.H. wrote the paper.

This work was supported by Swiss National Science Foundation Grants PP00A3-114923 and PP00P3-135438, the Clinical Research Priority Program "Sleep and Health" of the University of Zurich, and German Research Foundation Grant Wi 4059/1-1. We are grateful to Jan Born and Sabine Groch for their comments on a previous version of this manuscript.

The authors declare no competing financial interests.

Correspondence should be addressed to Reto Huber, Child Development Center, University Children's Hospital Zurich, Steinwiesstrasse 75, CH-8032 Zurich, Switzerland. E-mail: reto.huber@kispi.uzh.ch.

DOI:10.1523/JNEUROSCI.0962-14.2014

Copyright $\odot 2014$ the authors $\quad 0270-6474 / 14 / 3412568-08 \$ 15.00 / 0$
}

periments, better performance and greater neuronal changes were found in adults who started to exercise these cognitive abilities early in life than those who started later (Kuhl, 2010; Steele et al., 2013). However, the neurophysiological mechanisms of sensitive periods in humans remain essentially unknown. One open issue in this context is whether local processes of cortical maturation are directly linked to the sensitivity of a region to respond to specific learning experiences, as has been suggested previously (Penhune, 2011).

Importantly, experience-dependent plasticity is not restricted to the brain in the waking state but extends to the sleeping brain. An increase in synaptic density and efficacy has been shown to enhance neuronal synchronization, which in turn results in increased sleep slow-wave activity (SWA; Esser et al., 2007; Dash et al., 2009; Vyazovskiy et al., 2009; EEG activity at a frequency of $1-4.5 \mathrm{~Hz}$ that hallmarks deep slow-wave sleep). Specific learning experiences involving, for instance, intense visuomotor adaptation during the day resulted in an increase in post-learning SWA specifically over the cortical region that was shown to be involved during learning (Huber et al., 2004; Hanlon et al., 2009; Landsness et al., 2009; Määttä et al., 2010; Mascetti et al., 2013). Brain maturation is another factor that strongly affects sleep SWA. More specifically, not only has the SWA peak been found to parallel the posterior-to-anterior neurodevelopmental trajectory that has been described for gray matter volume, an anatomical marker of cortical maturation (Sowell et al., 2002; Casey et al., 
A

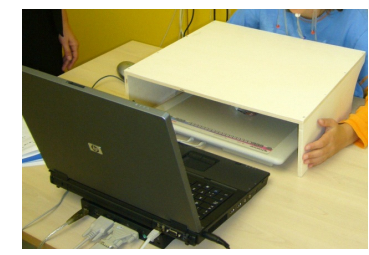

B

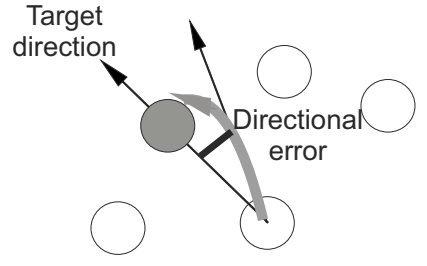

C

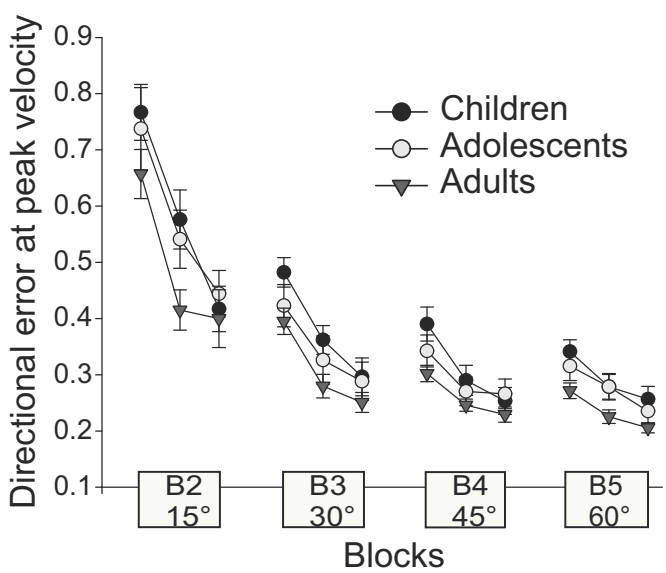

Figure 1. Visuomotor adaptation task and visuomotor performance in children, adolescents and adults. $\boldsymbol{A}$, During the visuomotor adaptation task, subjects sat in front of a screen that displayed a starting point in the middle of the screen and four possible target points. Subjects were required to move a cursor from the starting point to one of four targets while not seeing their movements because the hand was covered. Instead, they received a feedback about their movement on the screen. $\boldsymbol{B}$, As a measure of the quality of each movement, we computed the angle between the line from the initial hand position to the position of the target and the line to the position of the hand at the peak outward velocity (i.e., DEPV).C, To measure the time course of visuomotor adaptation, the DEPV was normalized by dividing it by the actual rotation angle. All three age groups were able to adapt to the imposed rotation as indicated by a decrease in DEPV within each block ( $p<0.001$ for main effect of trial).

2005), but SWA was also significantly correlated with gray matter volume (Kurth et al., 2010; Buchmann et al., 2011). In the present study, we investigated the following in children (9-11 years, $n=$ $15)$, adolescents ( $12-17$ years, $n=14)$, and adults ( $18-27$ years; $n=17$ ): (1) the effects of visuomotor adaptation on local SWA during the post-learning night; and (2) the association between measures of cortical maturation (i.e., gray matter volume and baseline SWA) and the local increase in SWA after visuomotor adaptation.

\section{Materials and Methods}

Subjects. Fifteen children aged 9-11 years (mean \pm SEM, $10.27 \pm 0.22$ years; six females, nine males), 14 adolescents aged $12-17$ years (15.51 \pm 0.36 ; two females, 12 males), and 17 adults ( $21.25 \pm 0.73$ years; three females, 14 males) participated in the study. Structural MRI data was available in 37 of these participants (children, $n=13$; adolescents, $n=$ 14 ; adults, $n=10$ ) because they had also participated in another experiment in which these measures were recorded (whole dataset was published previously by Buchmann et al., 2011). The participants were recruited via advertisements placed at the university, at the children's hospital, and in local newspapers. Interviews with the parents and children/adolescents and standardized questionnaires ensured that the participants had no behavioral problems, cognitive impairments, or sleep disorders. The subjects had no history of any neurological or psychiatric disorder and did not take any medication at the time of the experiment. The study was approved by the local ethics committee, and subjects gave written informed consent before participating. For the children and adolescents, this was accomplished by a parent. Additionally, all children and adolescents provided verbal assent.

Procedure. Each subject participated in two conditions (baseline and adaptation) according to a within-subject crossover design, with the order of conditions balanced across subjects. The two conditions for each participant were separated by at least 1 week. In both experimental conditions, subjects came to the sleep laboratory $\sim 3 \mathrm{~h}$ before subjects' habitual bedtime. The high-density electrodes net was placed immediately thereafter. In each condition, subjects performed on a visuomotor task in the evening and went to bed thereafter.

Visuomotor adaptation task. To investigate local changes in sleep SWA, we chose the visuomotor adaptation task because it is well known to involve a specific region over the right parietal cortex (Ghilardi et al., 2000). This task requires the subjects to move a cursor from a starting point to one of four possible targets, thereby receiving a visual feedback about their hand movements on the screen in front of them (Fig. $1 A)$. The experimenter permanently stood next to the subject during task performance to intervene in the case of a performance decrease as a result of lack of motivation or changes in posture (as a possible strategy to deal with the imposed rotation). Compared with previous experiments in adults (Huber et al., 2004; Landsness et al., 2009; Määttä et al., 2010), we decided to use an easier version of the visuomotor adaptation task with only four instead of eight possible targets to make this task feasible for children. This was based on the following: (1) previous findings demonstrating that implicit motor learning was lower in children compared with adults (Fletcher et al., 2000; Thomas et al., 2004; Wilhelm et al., 2008); (2) on our own pilot data showing a high rate of dropouts in the learning session when using the eight-target task version; and (3) the fact that a four-target version of the task has been successfully used in almost all of the previous studies in children (Ferrel et al., 2001; Contreras-Vidal et al., 2005). With the reduction to four targets, we intended to reduce the length of the test period (to avoid an increase in tiredness and a decrease in motivation at the end of the blocks) and the difficulty of the task. In one of two conditions (i.e., the adaptation condition), the feedback was rotated at a fixed angle. Subjects performed on a total of seven blocks (B1-B7) each, including three trials of the same rotation angle with all of them being separated by a short break. In each of the trials, subjects had to execute 44 movements to one of the four targets in a pseudorandomized order. To familiarize the subjects to the requirements of the task, the test session started with one block without any feedback rotation (B1). Thereafter, feedback was rotated at a blockwise increasing angle (B2, 15 $\left.\mathrm{B} 3,30^{\circ} ; \mathrm{B} 4,45^{\circ} ; \mathrm{B} 5,60^{\circ}\right)$. Actual visuomotor performance was tested in a separate test block at the end of the test session (B7). Preceding this test block, a block of $0^{\circ}$ rotation (B6) was introduced to wash out any residual rotation of the internal model. We computed the directional error for each movement as the angle between the line from the initial hand position to the position of the target and the line to the position of the hand at the peak outward velocity [directional error at peak velocity (DEPV); Fig. $1 \mathrm{~B}$ ]. As a measure of the final performance level in the adaptation condition, we calculated the average performance across all 44 movements in the last trial of B7. In the baseline condition, subjects had to execute the same amount of hand movements from the starting point to one of four targets as in the adaptation condition, but feedback was not rotated at any time. We chose exactly this kind of task to reduce the possibility that the three age groups can use different strategies. Previous experiments have uncovered two possible strategies that can be used in such a visuomotor adaptation task, i.e., explicit and implicit strategies (Benson et al., 2011; Taylor and Ivry, 2012; Taylor et al., 2014). However, the spontaneous generation of explicit knowledge in a visuomotor adaptation task, without any additional task instructions given by the experimenter, depends on specific features that were not present in our task. These conditions include the following: (1) sufficient amount of rest between trials, which allows for movement corrections and the conscious reflection of possible sources of performance errors (in our study, targets were presented one after another at an interval of $1 \mathrm{~s}$, which forced the subjects to quickly execute the movements); and (2) the abrupt imposing of the final rotation, which produces a large error signal and thereby creates a situation in which subjects might become aware of the perturbation (the rotation was gradually imposed in our study). Importantly, even if a protocol fulfils these two requirements, the actual number of 
subjects that gain insight into the nature of the imposed perturbation and therefore are able to use an explicit strategy to improve their performance is extremely low [Benson et al. (2011) found only 3 of 27 adult subjects]. Accordingly, it is very unlikely that the three age groups differed with regard to the use of their strategy because, with this version of the task, it was nearly impossible to generate any explicit knowledge that would be the bases for using an explicit strategy.

EEG recording, preprocessing, and analysis. All-night sleep EEG, electrooculogram, and electromyogram were recorded in the sleep laboratory of the University Children's Hospital Zürich (Zürich, Switzerland). All participants were monitored during both experimental nights using high-density sleep EEG (Electrical Geodesics Sensor Net for long-term monitoring, 128 channels, referenced to a vertex electrode for direct visualization and to the average across all channels for data analysis). Data were sampled at $500 \mathrm{~Hz}(0.01-200 \mathrm{~Hz})$. Offline, the EEG was bandpass filtered $(0.5-50 \mathrm{~Hz})$ and downsampled to $128 \mathrm{~Hz}$. Artifacts were rejected on a $20 \mathrm{~s}$ basis after visual inspection and if power exceeded a threshold based on a mean power value in the $0.75-4.5$ and $20-30 \mathrm{~Hz}$ bands (Huber et al., 2000). Poor-quality EEG channels were excluded (mean \pm SEM number of excluded channel, $2.01 \pm 0.22$; range, $0-12$ ), and, for the topographical analyses, data of excluded channels were interpolated by the method of spherical linear interpolation. All additional analyses were based on re-referenced data: for every EEG sample, the value of each channel was divided by the average value across all 109 channels above the ears. The EEG was visually scored for sleep stages at frontal, central, and occipital electrodes (20 s epochs) based on American Academy of Sleep Medicine standard criteria (Iber et al., 2007). For qualitative exploration, spectral analysis was performed for all channels [fast Fourier transform routine, Hanning window, $20 \mathrm{~s}$ epochs (averages of five $4 \mathrm{~s}$ epochs), frequency resolution of $0.25 \mathrm{~Hz}$ ]. The $20 \mathrm{~s}$ spectral power values were then averaged for a certain time window. Subsequent analyses were restricted to the slow-wave frequency band (SWA; $1-4.5 \mathrm{~Hz}$ ). Because we focused on experience-dependent local SWA changes, SWA was normalized by dividing SWA at a single electrode by the average SWA over all 109 electrodes as done previously (Huber et al., 2004). Absolute SWA (i.e., without normalization) is subject to profound dayto-day changes, which makes this measure less sensitive to uncover small experience-dependent local changes in SWA. Nevertheless, we also analyzed experience-dependent changes in absolute SWA and found the same effects that were, as expected, less pronounced compared with the normalized data. We defined as a cluster of electrodes at least seven significant neighboring electrodes. We chose this number of electrodes because, when calculating 109 statistical tests, six electrodes will become significant by chance given a significance level of $5 \%$ (which means that the calculation of 100 statistical tests will lead to five significant results without any real difference between the conditions). Note that this threshold is conservative because the likelihood of these six electrodes being neighboring electrodes is much lower.

Structural MRI and surface-based analysis. All images were obtained on the same $3 \mathrm{~T}$ scanner (a GE Healthcare Signa HDx). We used a T1weighted gradient-echo whole-brain image, repetition time of $8.928 \mathrm{~ms}$, echo time of $3.496 \mathrm{~ms}$, and flip angle of $13^{\circ}$; image resolution in the $x-y-z$ direction was $256 \times 256 \times 140$ voxels, resulting in a resolution of $0.938 \times$ $0.938 \times 1.2 \mathrm{~mm}$.

Parietal gray matter volumes were calculated using Freesurfer stable version 4.5.0 for Mac OS 10.5.2 (http://surfer.nmr.mgh.harvard.edu; Dale et al., 1999; Fischl et al., 1999). In this software, the T1 images are analyzed using the recon-all procedure, which treats subcortical structures and cortical hemispheres separately. The hemispheres are registered with a spherical atlas, which uses individual cortical folding patterns to match cortical geometry across subjects. This procedure allows parcellation of the cerebral cortex into gyral and sulcal units. It has been shown that Freesurfer statistics are robust against white noise in the images and that results are similar for multiple T1 images and one T1 image (Jovicich et al., 2009). Areas from the surface-based analysis were fused to form the left and right parietal lobes.

Statistical analyses. Statistical analysis of DEPV was based on a $4 \times 3 \times$ 3 ANOVA, including the group factor "age group" (children, adolescents, and adults) and the repeated-measures factors "block", represent- ing the four blocks of gradual adaptation, and "trial" (three trials of the same rotation within each block). Analyses of experience-dependent local changes in SWA were based on a $2 \times 3$ ANOVA, including the repeated-measures factor "condition" (baseline vs adaptation) and the group factor "age group". In case of a significant main effect of age group in these ANOVAs, post hoc comparisons using Tukey's HSD were calculated to further elucidate the effect of age group. In case of a significant interaction including the factor age group, separate post hoc $t$ tests were calculated. Correlation analyses were conducted using Pearson's correlation coefficient. The level of significance was set to $p=0.05$.

\section{Results}

\section{Visuomotor performance before sleep}

In the adaptation condition, subjects performed on seven blocks, each including three trials of the same rotation angle, with all blocks being separated by a short break. In each of the trials, subjects had to execute 44 movements to one of four targets. After one block without feedback rotation (B1), feedback was rotated at a blockwise increasing angle in the subsequent blocks (B2, 15 ${ }^{\circ}$; $\left.\mathrm{B} 3,30^{\circ} ; \mathrm{B} 4,45^{\circ} ; \mathrm{B} 5,60^{\circ}\right)$. All three age groups were able to adapt to the imposed rotation, as indicated by a decrease in the DEPV from B2 to B5 and across the three trials of the same rotation in each block (main effect of block, $F_{(1.7,68.1)}=135.85, p<0.001$; main effect of trial, $F_{(1.8,77.5)}=244.52, p<0.001$; Fig. $1 C$ ). In the final trial of B7 (the last 44 movements during the test session), adults outperformed children (mean \pm SEM DEPV: adults, $12.76 \pm 0.97$; children, $18.6 \pm 1.48$; difference between both age groups, $p=0.026$ ), whereas no difference was found between children and adolescents (adolescents, $17.96 \pm 2.21 ; p>0.95$ ).

\section{Experience-dependent local changes in SWA}

In both conditions, high-density EEG (128 electrodes) was recorded during the night. As expected, children, adolescents, and adults differed significantly with respect to a number of sleep parameters (i.e., total sleep time, amount of sleep stages 2 and 3). Importantly, there were no differences in any sleep parameter when comparing baseline with adaptation condition (for sleep parameters and statistics, see Table 1).

To investigate whether visuomotor adaptation affects local SWA, we calculated for each of the 109 electrodes a $2 \times 3$ ANOVA, including the within subject-factor condition (adaptation/baseline) and the between-subject factor age group (children/adolescents/adults). During the post-learning night, SWA was increased in a cluster of eight electrodes that were located over the right parietal cortex (main effect of condition for each of these electrodes, $p<0.05$; Fig. $2 A$ ). To further investigate the modulating affect of age on experience-dependent changes in SWA, we averaged SWA over these eight electrodes and calculated a 2 (condition) $\times 3$ (age group) ANOVA. This analysis revealed that the effect of visuomotor adaptation on local SWA was critically modulated by the subjects' age (main effect of condition, $F_{(1,43)}=13.63, p<0.001$; interaction of condition $\times$ age group, $\left.F_{(2,43)}=3.50, p=0.039\right)$. Post hoc $t$ tests indicated that visuomotor adaptation strongly affects SWA in the parietal cluster in the group of children (adaptation vs baseline, $p=0.003$ ) but not in adolescents $(p=0.20)$ and adults $(p=0.36$; Fig. $2 B)$. Accordingly, age was negatively correlated with the local increase in SWA $(r=-0.35, p=0.018$; Fig. $2 C)$.

To analyze the time course of experience-dependent local changes in SWA across the night, we calculated SWA in the parietal cluster in the first 30 min of non-rapid eye movement (REM) sleep in each of the first five sleep cycles in both experimental conditions. In children, higher SWA in the parietal cluster was found after visuomotor adaptation compared with baseline in the 
Table 1. Sleep in both experimental conditions in all three age-groups

\begin{tabular}{|c|c|c|c|c|c|c|c|}
\hline & Children & & Adolescents & & Adults & & \\
\hline & Baseline & Adaptation & Baseline & Adaptation & Baseline & Adaptation & $p$ value \\
\hline TST (min) & $524.2 \pm 11.7$ & $533.1 \pm 8.7$ & $449.1 \pm 20.0$ & $466.7 \pm 14.4$ & $436.7 \pm 12.1$ & $440.4 \pm 9.4$ & 0.017 \\
\hline Sleep latency (min) & $26.0 \pm 3.1$ & $22.8 \pm 3.4$ & $15.9 \pm 2.3$ & $14.7 \pm 2.5$ & $21.3 \pm 2.6$ & $17.8 \pm 2.0$ & 0.02 \\
\hline Vigilance states in \% & & & & & & & \\
\hline Wake & $54.0 \pm 10.2$ & $34.3 \pm 9.0$ & $20.8 \pm 4.8$ & $34.7 \pm 10.4$ & $34.1 \pm 8.4$ & $24.3 \pm 4.4$ & 0.20 \\
\hline Stage 1 & $7.5 \pm 0.8$ & $6.7 \pm 0.7$ & $8.2 \pm 1.4$ & $7.1 \pm 0.8$ & $5.8 \pm 0.5$ & $6.3 \pm 0.6$ & 0.26 \\
\hline Stage 2 & $45.6 \pm 2.1$ & $49.1 \pm 1.7$ & $50.4 \pm 1.4$ & $51.6 \pm 1.6$ & $55.5 \pm 1.7$ & $54.4 \pm 1.5$ & 0.004 \\
\hline SWS & $27.6 \pm 2.2$ & $24.6 \pm 2.1$ & $22.5 \pm 1.5$ & $21.6 \pm 1.8$ & $18.6 \pm 2.2$ & $19.6 \pm 2.0$ & 0.04 \\
\hline REM sleep & $19.3 \pm 1.0$ & $19.5 \pm 1.0$ & $18.9 \pm 1.5$ & $19.6 \pm 0.7$ & $19.9 \pm 1.2$ & $19.8 \pm 1.1$ & 0.90 \\
\hline
\end{tabular}

Mean \pm SEM total sleep time (TST) and sleep latency, i.e., the first occurrence of stage 2 sleep, determined with reference to the time of lights off and time spent awake, in stage 1 sleep, stage 2 sleep, slow wave sleep (SWS), and REM sleep in percentage of TST. The $p$ values for the main effect of age group in 2 (condition) $\times 3$ (age group) ANOVAs are shown in the last column (main effect of condition for all analyses, $p>0.12$ ).

A

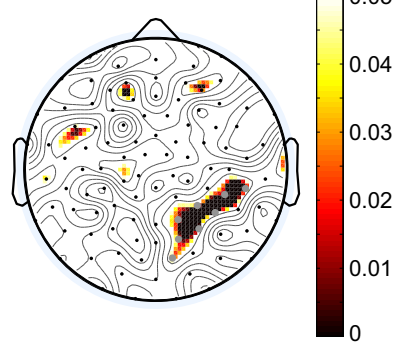

B

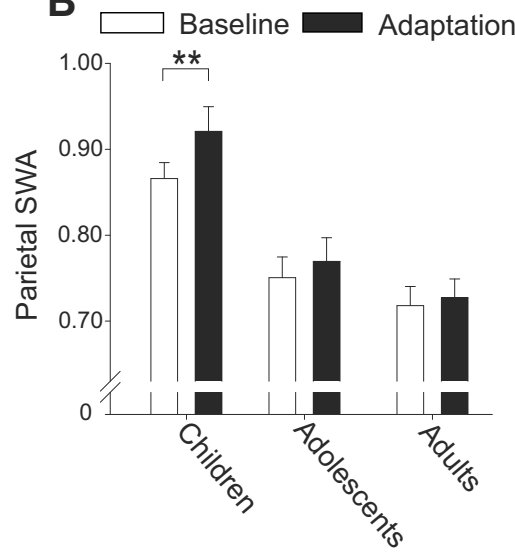

C

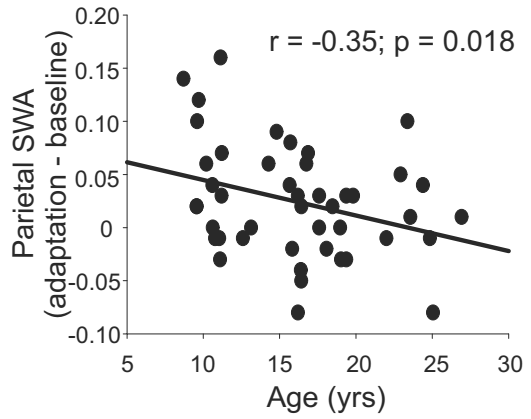

Figure 2. Local changes in SWA after visuomotor adaptation and the modulating affect of age. $A$, After visuomotor adaptation, SWA was increased in a cluster of eight electrodes located over the right parietal cortex [all electrodes marked as gray dots were $p<0.05$ for main effect condition (adaptation vs baseline)]. $\boldsymbol{B}$, Mean \pm SEM of SWA in the eight electrodes of the parietal cluster was significantly higher in the adaptation condition (black bars) compared with the baseline condition (white bars) in the group of children $(p=0.003$ ) but not in adolescents and adults (both $p>0.20$ ). C, Age (in years) was negatively correlated with the increase in SWA (i.e., the difference in SWA between the adaptation and baseline condition) in the parietal cluster after visuomotor adaptation. ${ }^{* *} p<0.01$.

A

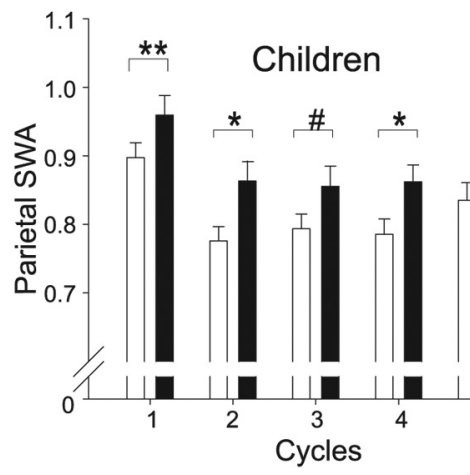

B

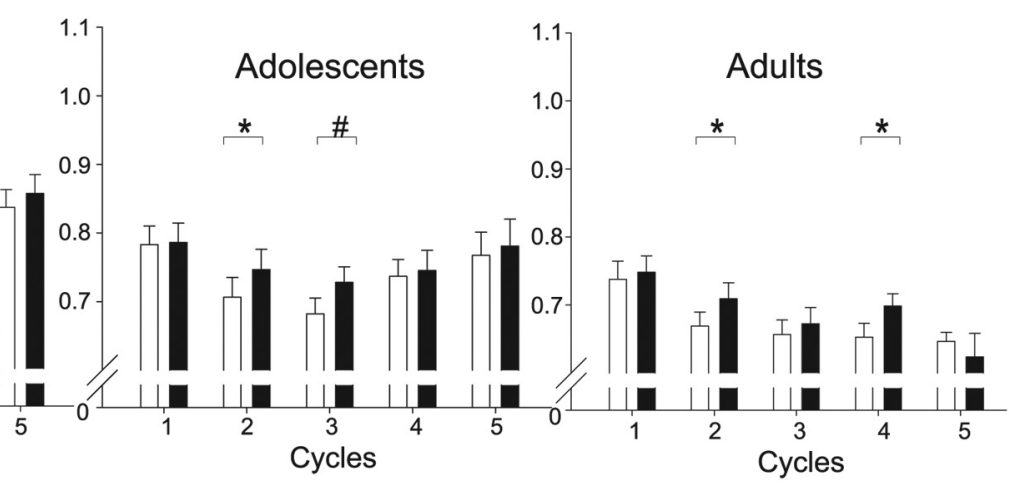

Figure 3. Time course of experience-dependent SWA changes across the night in all three age groups. Mean \pm SEM of SWA in the eight electrodes of the parietal cluster in the first 30 min of non-REM sleep of the first five cycles in the adaptation (black bars) and baseline (white bars) conditions in children $(\boldsymbol{A})$, adolescents $(\boldsymbol{B})$, and adults $(\boldsymbol{C}) .{ }^{*} p<0.10 ;{ }^{*} p<0.05 ;{ }^{* *} p<0.01$.

first four cycles (first, second, and fourth, $p<0.05$; third, $p<$ 0.10 ; Fig. $3 A$ ), whereas in adolescents and adults, this was the case in two of five cycles (adolescents: second, $p<0.05$ and third, $p<$ 0.10 ; adults: second and fourth, $p<0.05$; Fig. $3 B, C$; for the topoplots indicating local changes in SWA in the first four cycles in each of the three age groups, see Fig. 4).

In a next step, we tested whether the observed age-dependent differences in experience-dependent SWA changes are directly related to processes of cortical maturation. SWA is a valid marker of brain maturation because it was shown to be highest in regions undergoing cortical reorganization during maturation (Kurth et al., 2010; Buchmann et al., 2011). We used SWA in the first 30 min of non-REM sleep in the baseline condition as a measure of cortical maturation. In line with previous imaging studies showing a posterior-to-anterior trajectory (Sowell et al., 2002; Casey et al., 2005), we found that the SWA peak shifted from occipital to frontal areas from childhood to adolescence (Fig. 5A). Moreover, baseline SWA in the eight electrodes of interest (i.e., those elec- 


\section{Children Adolescents Adults}
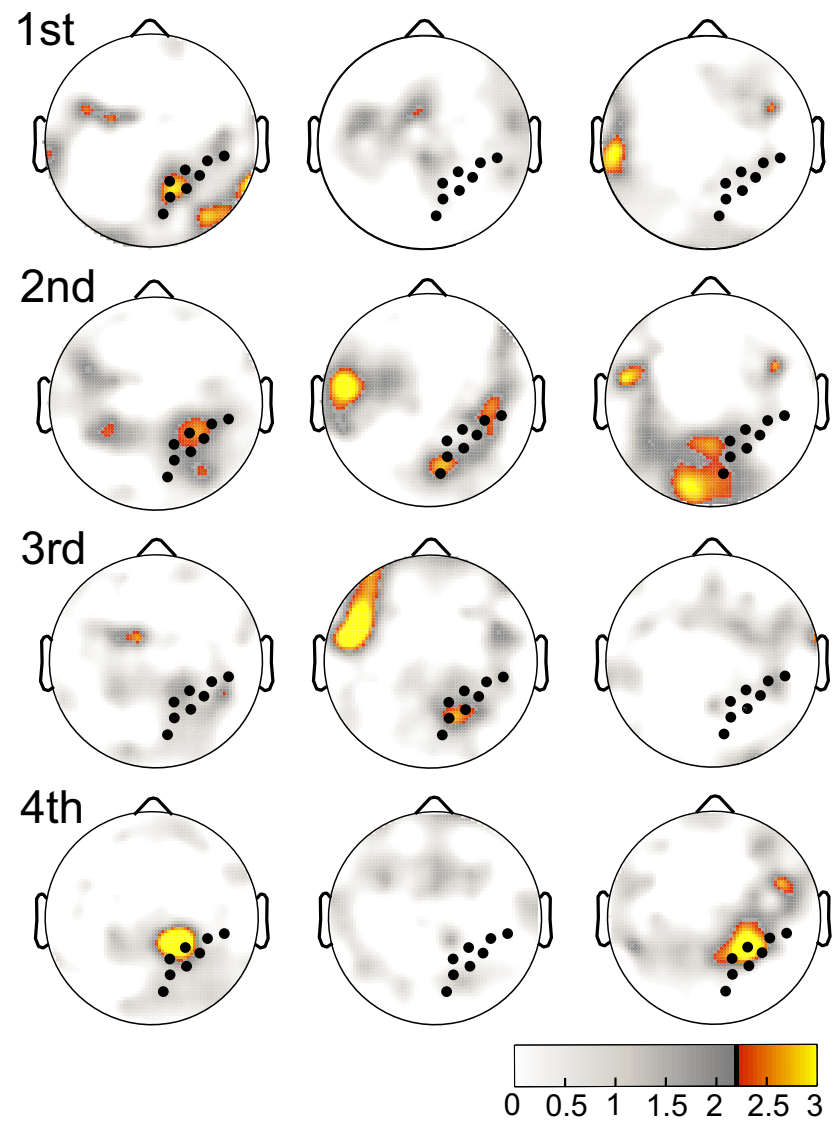

Figure 4. Local changes in SWA after visuomotor adaptation in all three age groups for the first $30 \mathrm{~min}$ in each of the first four sleep cycles. $t$ values from 0 to 3 are presented for all electrodes. Regions of significantly increased SWA are shown from red to yellow (the critical $t$ value in all age groups and cycles of 2.15 is marked by the black line in the color bar). Note that the right parietal cluster (as indicated by the black dots) is the one that most consistently showed a significant increase after visuomotor adaptation. The discussion of regions becoming significant in single cycles and age groups (e.g., the frontocentral region in the second and third cycles in adolescents) is interesting but goes beyond the scope of this study.

trodes over the parietal cortex that had been found to show increased SWA after visuomotor adaptation) was highest in the group of children (main effect of age group, $F_{(2,42)}=17.87, p<$ 0.001 ; children vs adults, $p<0.001$; children vs adolescents, $p<$ 0.01 ; adolescents vs adults, $p=0.58$; Fig. $5 B$ ). Importantly, baseline SWA in this cluster was significantly correlated with the increase in SWA after visuomotor adaptation in children, whereas in adults or adolescents, such a correlation was not found (children, $r=0.70, p=0.004$; adolescents, $r=-0.06, p=0.85$; adults, $r=-0.26, p=0.29$; Fig. $5 C$ ). To verify that baseline SWA can indeed be used as a marker of maturation in the current study sample, we analyzed structural MRI markers of brain maturation in the region of interest (i.e., the right parietal lobe) in a subset of the study sample ( $n=37$; children, $n=13$; adolescents, $n=14$; adults, $n=10$ ). Gray matter volume in the right parietal lobe was significantly correlated with baseline SWA in the cluster of interest over the right parietal lobe $(r=0.46, p=0.004$; Fig. $5 D)$. Moreover, gray matter volume in the right parietal lobe correlated with the increase in SWA after visuomotor adaptation in the right parietal electrode cluster $(r=0.46, p=0.004$; age corrected, $r=0.35, p=0.034$; Fig. $5 E$ ). A lack of a correlation between gray matter volume in the left parietal lobe and the SWA increase in the right parietal cluster $(r=0.27, p=$ 0.11 ; age corrected, $r=0.11, p=0.54$ ) points toward an effect that is specific for the region that is involved in the task rather than a general effect of brain maturation.

\section{Discussion}

We subjected children, adolescents, and adults to a novel learning experience (i.e., a visuomotor learning task) that has been shown previously to activate circumscribed brain regions in a PET study (Brodmann areas 40 and 7, which are located in the right parietal cortex; Ghilardi et al., 2000). In the post-learning night, we observed a gain in SWA in a cluster of eight electrodes over the right parietal scalp that was strongest in children compared with adolescents and adults. The finding of increased local SWA in a region that is known to be recruited during task performance is in line with a number of previous experiments in humans and rodents reporting a link between learning experiences during the wake phase and local SWA during subsequent sleep (Kattler et al., 1994; Huber et al., 2004, 2006; Hanlon et al., 2009; Mascetti et al., 2013). SWA in the surface EEG reflects a highly synchronous alternating pattern of neuronal firing (depolarization) and neuronal silence (hyperpolarization) in large neuronal networks (Steriade et al., 1993). Novel learning can lead to the formation and selective elimination of synapses and the modulation of synaptic strength (for review, see Fu and Zuo, 2011). An increase in synaptic density and efficacy has been shown to enhance neuronal synchronization, which in turn results in greater SWA (Esser et al., 2007; Dash et al., 2009; Vyazovskiy et al., 2009). Thus, it is reasonable to assume that the local change in SWA in the right parietal cluster in the post-learning night reflects the increase in synaptic strength and/or density in this specific region induced by the learning task.

Our finding of greatest changes in local SWA in the group of children indicates that experience-dependent plasticity is highest during childhood and declines thereafter. These data are well in line with a number of previous findings pointing toward the existence of sensitive periods during human development for specific skills, such as second-language learning and musical training (Elbert et al., 1995; Schlaug et al., 1995; Nicholas and Geers, 2007; Bailey and Penhune, 2010; Kuhl, 2010; Steele et al., 2013). In most of these experiments, better performance was found in adults who started to train these cognitive abilities early in life when compared with those who started later. As discussed previously (Penhune, 2011), these studies can only give indirect information on the modulating effect of age on experiencedependent plasticity because they are subject to multiple confounding factors. More specifically, one can hardly exclude that children ("early learners") and adults ("late learners") already differ with regard to preexisting factors, such as genetically determined differences in skill or individual differences in motivation and training. These confounding factors were eliminated in our study by mapping local changes in SWA immediately after learning. Importantly, using this approach we were able to show that sensitive periods for visuomotor learning exist during human development.

The neurophysiological mechanisms underlying sensitive periods in humans are not well understood. It has been argued recently that learning a specific skill induces profound and longlasting effects in the brain and behavior at the time during which the involved cortical region undergoes maturation (Penhune, 2011; Steele et al., 2013). Maturation does not occur simultaneously in different brain regions, but motor and sensory systems mature earliest, temporal and parietal association cortices mature 
A

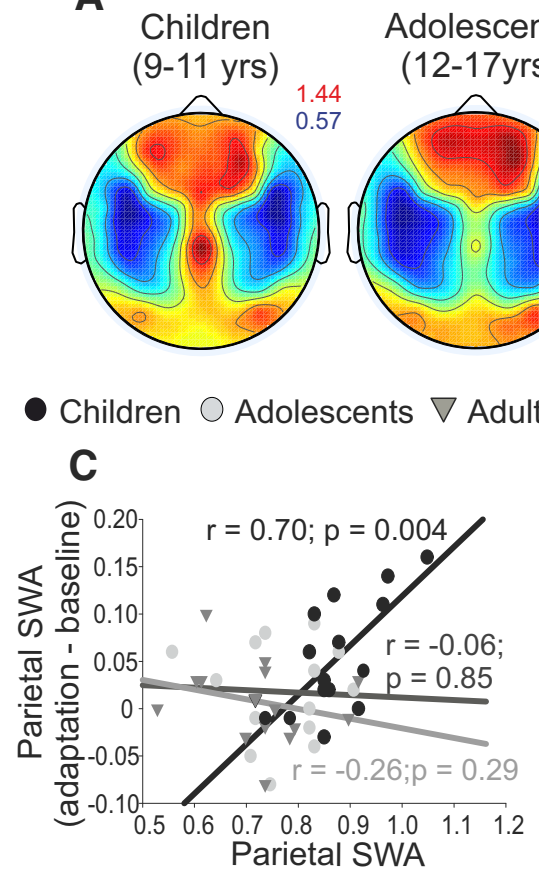

(baseline)
D

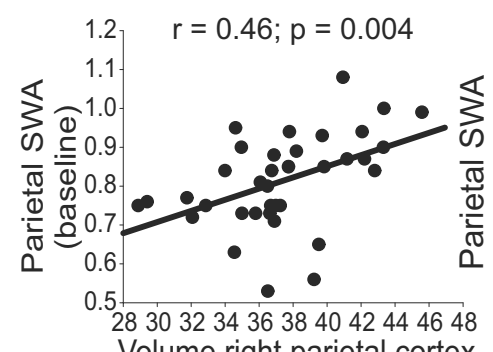

Volume right parietal cortex

(in $\mathrm{cm}^{3}$ )
B
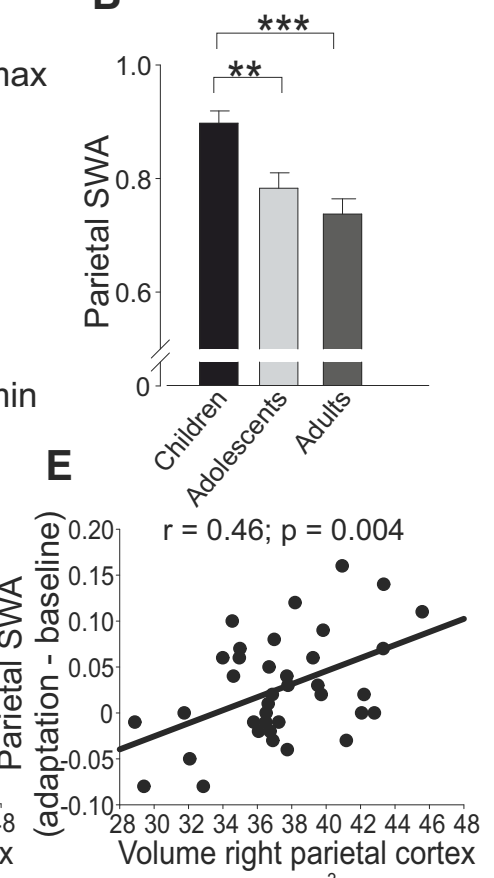

(in $\mathrm{cm}^{3}$ )

Figure 5. SWA topography, baseline SWA in the parietal cluster, and local increase in SWA after visuomotor adaptation. $A$, Topography of SWA in the first 30 min of non-REM sleep in children, adolescents, and adults in the baseline condition. SWA in the parietal cluster recorded in the baseline condition was highest in children $\left({ }^{* *} p<0.01\right.$; $\left.{ }^{* * *} p<0.001\right)(\boldsymbol{B})$, and it was significantly correlated with the increase in SWA after visuomotor adaptation in children but not in adults and adolescents (C).D, A significant correlation was found between gray matter volume in the right parietal lobe and baseline SWA in the cluster of interest over the right parietal lobe $(r=0.46, p=0.004)$. $E$, Gray matter volume in the right parietal lobe was correlated with the increase in SWA after visuomotor adaptation in the right parietal electrode cluster $(r=0.46, p=0.004)$.

next, and prefrontal and lateral temporal regions mature latest (Sowell et al., 2002; Gogtay et al., 2004; Casey et al., 2005). Consequently, adolescents and adults in our study may have shown lower experience-dependent plastic changes after visuomotor adaptation than children because their parietal cortex is already fully mature. Our findings on a strong correlation between gray matter volume in the right parietal cortex and the local experience-dependent increase in SWA over the right parietal scalp are in line with this notion. Moreover, SWA over the parietal cluster, which can be used as an electrophysiological marker of the maturation of this region (Kurth et al., 2010, 2012; Buchmann et al., 2011), was also significantly associated with the experience-dependent increase in SWA over this region. These findings suggest the following: (1) actual processes of brain maturation within a specific region favor experience-dependent plasticity in this region; and (2) mapping local SWA during postlearning sleep is a sensitive tool to uncover this relationship. This suggestion is supported by an animal experiment that studied the effect of sensory experiences on SWA in young and old mice and cats (Miyamoto et al., 2003). In this study, visual deprivation (i.e., dark rearing) of young mice and cats during the critical period for ocular dominance resulted in a dramatic reduction of SWA over the primary visual cortex, whereas this effect was not observable in older animals. Future studies need to elucidate the exact time courses of experience-dependent plasticity for a variety of cognitive tasks that depend on different brain regions. An estimation of the developmental time window in which the brain is most vulnerable to the external input of specific experiences (i.e., language learning or the acquisition of motor skills) can have important implications not only for educational policy but also clinical therapy.

SWA is not only an indicator for experience-dependent plasticity but is itself causally related to the long-term storage of newly acquired experiences. For example, task performance at a later recall was improved when SWA during post-learning sleep had been boosted by auditory or electrical stimulation (Marshall et al., 2006; Ngo et al., 2013). Thus, current models suggest a cycle of novel learning experiences, leading to a local increase of SWA during sleep, which in turn results in a strengthening and/or improvement of performance in the learned task after sleep (Nere et al., 2013; Rasch and Born, 2013). Whether visuomotor adaptation indeed develops optimally over time in children and whether this is related to their strong increase in local SWA after visuomotor adaptation needs to be studied in future experiments.

Moreover, SWA has been hypothesized recently to be actively engaged in processes of brain maturation (Campbell and Feinberg, 2009; Huber and Born, 2014). Brain maturation is a process of intense cortical reorganization characterized by a rapid increase in synaptic density, followed by an elimination of synapses (for review, see Rakic et al., 1994). In adolescent mice, the production of synapses was found to most efficiently occur during wakefulness, whereas the elimination of synapses was most prominent during sleep (Maret et al., 2011). Here, we found that stimulating a specific brain region by a learning task at the developmental time of high synaptic density (as indicated by gray matter density and baseline SWA) results in a strong increase in SWA in the same region. This increase in SWA presumably reflects the learning-dependent increase in synaptic density/ strength, which may lead to a more efficient reorganization of the involved cortical circuits. Together with previous work revealing the importance of sleep for brain plasticity during development in animals (Frank et al., 2001; Jha et al., 2005), the current experiment indicates that studying processes during sleep may well be fruitful to improve our understanding of how experiences effect the brain during early development. Moreover, if indeed sleep is 
centrally involved in shaping our brain during development, disturbed or insufficient sleep during development might have negative consequences on cortical maturation.

A sensitive period within which an experience can induce major changes in the brain does not exclude that the same experience has no affect after closure of this window. Previous studies indeed reported an increase of SWA over the parietal cortex after visuomotor adaptation in a group of adults (Huber et al., 2004; Landsness et al., 2009). The weaker effect of visuomotor adaptation on SWA in our adults compared with these previous experiments can be attributed to our specific task properties (i.e., four instead of eight targets; for details, see Materials and Methods). This might have resulted in less intense learning, which in turn led to less plastic changes in the adults' brain. In adults, because of their decreased level of brain plasticity, more intense stimulation of neuronal networks might be needed to reach a substantial level of experience-dependent changes. This interpretation is in line with a previous experiment in adults demonstrating that the difficulty of a task determines whether learning induces SWA changes in the subsequent night (Schmidt et al., 2006).

An obvious limitation of the study is its cross-sectional nature. Subjects from different cohorts might differ with regard to individual variables (e.g., experience in using a computer mouse), and one might ask whether such cohort differences can have confounded our findings. If this was the case, learning performance should have differed between the age groups, with children performing better than adolescents and adults. We found only very subtle behavioral differences with children performing worse than adults at the end of learning, whereas children and adolescents did not differ. Thus, it is unlikely that cohort-specific differences affecting learning performance can explain the observed age-dependent differences in local SWA changes after visuomotor adaptation. Nevertheless, additional studies are needed in which subgroups of the same cohort are tested at three different ages to fully exclude this possible confound.

\section{References}

Bailey JA, Penhune VB (2010) Rhythm synchronization performance and auditory working memory in early- and late-trained musicians. Exp Brain Res 204:91-101. CrossRef Medline

Benson BL, Anguera JA, Seidler RD (2011) A spatial explicit strategy reduces error but interferes with sensorimotor adaptation. J Neurophysiol 105: 2843-2851. CrossRef Medline

Buchmann A, Ringli M, Kurth S, Schaerer M, Geiger A, Jenni OG, Huber R (2011) EEG sleep slow-wave activity as a mirror of cortical maturation. Cereb Cortex 21:607-615. CrossRef Medline

Campbell IG, Feinberg I (2009) Longitudinal trajectories of non-rapid eye movement delta and theta EEG as indicators of adolescent brain maturation. Proc Natl Acad Sci U S A 106:5177-5180. CrossRef Medline

Casey BJ, Tottenham N, Liston C, Durston S (2005) Imaging the developing brain: what have we learned about cognitive development? Trends Cogn Sci 9:104-110. CrossRef Medline

Contreras-Vidal JL, Bo J, Boudreau JP, Clark JE (2005) Development of visuomotor representations for hand movement in young children. Exp Brain Res 162:155-164. CrossRef Medline

Dale AM, Fischl B, Sereno MI (1999) Cortical surface-based analysis. I. Segmentation and surface reconstruction. Neuroimage 9:179-194. CrossRef Medline

Dash MB, Douglas CL, Vyazovskiy VV, Cirelli C, Tononi G (2009) Longterm homeostasis of extracellular glutamate in the rat cerebral cortex across sleep and waking states. J Neurosci 29:620-629. CrossRef Medline

Elbert T, Pantev C, Wienbruch C, Rockstroh B, Taub E (1995) Increased cortical representation of the fingers of the left hand in string players. Science 270:305-307. CrossRef Medline

Esser SK, Hill SL, Tononi G (2007) Sleep homeostasis and cortical synchronization: I. Modeling the effects of synaptic strength on sleep slow waves. Sleep 30:1617-1630. Medline
Ferrel C, Bard C, Fleury M (2001) Coordination in childhood: modifications of visuomotor representations in 6- to 11-year-old children. Exp Brain Res 138:313-321. CrossRef Medline

Fischl B, Sereno MI, Dale AM (1999) Cortical surface-based analysis. II: Inflation, flattening, and a surface-based coordinate system. Neuroimage 9:195-207. CrossRef Medline

Fletcher J, Maybery MT, Bennett S (2000) Implicit learning differences: a question of developmental level? J Exp Psychol Learn Mem Cogn 26:246252. CrossRef Medline

Frank MG, Issa NP, Stryker MP (2001) Sleep enhances plasticity in the developing visual cortex. Neuron 30:275-287. CrossRef Medline

Fu M, Zuo Y (2011) Experience-dependent structural plasticity in the cortex. Trends Neurosci 34:177-187. CrossRef Medline

Ghilardi M, Ghez C, Dhawan V, Moeller J, Mentis M, Nakamura T, Antonini A, Eidelberg D (2000) Patterns of regional brain activation associated with different forms of motor learning. Brain Res 871:127-145. CrossRef Medline

Gogtay N, Giedd JN, Lusk L, Hayashi KM, Greenstein D, Vaituzis AC, Nugent TF 3rd, Herman DH, Clasen LS, Toga AW, Rapoport JL, Thompson PM (2004) Dynamic mapping of human cortical development during childhood through early adulthood. Proc Natl Acad Sci U S A 101:8174-8179. CrossRef Medline

Hanlon EC, Faraguna U, Vyazovskiy VV, Tononi G, Cirelli C (2009) Effects of skilled training on sleep slow wave activity and cortical gene expression in the rat. Sleep 32:719-729. Medline

Huber R, Born J (2014) Sleep, synaptic connectivity, and hippocampal memory during early development. Trends Cogn Sci 1281:1-12. CrossRef Medline

Huber R, Graf T, Cote KA, Wittmann L, Gallmann E, Matter D, Schuderer J, Kuster N, Borbély AA, Achermann P (2000) Exposure to pulsed highfrequency electromagnetic field during waking affects human sleep EEG. Neuroreport 11:3321-3325. CrossRef Medline

Huber R, Ghilardi MF, Massimini M, Tononi G (2004) Local sleep and learning. Nature 430:78-81. CrossRef Medline

Huber R, Ghilardi MF, Massimini M, Ferrarelli F, Riedner BA, Peterson MJ, Tononi G (2006) Arm immobilization causes cortical plastic changes and locally decreases sleep slow wave activity. Nat Neurosci 9:1169-1176. CrossRef Medline

Iber C, Ancoli-Israel S, Chesson AL, Quan SF, eds (2007) The AASM manual for the scoring of sleep and associated events: rules, terminology and technical specifications, Ed 1. Westchester, IL: American Academy of Sleep Medicine.

Jha SK, Jones BE, Coleman T, Steinmetz N, Law CT, Griffin G, Hawk J, Dabbish N, Kalatsky VA, Frank MG (2005) Sleep-dependent plasticity requires cortical activity. J Neurosci 25:9266-9274. CrossRef Medline

Jovicich J, Czanner S, Han X, Salat D, van der Kouwe A, Quinn B, Pacheco J, Albert M, Killiany R, Blacker D, Maguire P, Rosas D, Makris N, Gollub R, Dale A, Dickerson BC, Fischl B (2009) MRI-derived measurements of human subcortical, ventricular and intracranial brain volumes: reliability effects of scan sessions, acquisition sequences, data analyses, scanner upgrade, scanner vendors and field strengths. Neuroimage 46:177-192. CrossRef Medline

Kattler H, Dijk DJ, Borbély AA (1994) Effect of unilateral somatosensory stimulation prior to sleep on the sleep EEG in humans. J Sleep Res 3:159164. CrossRef Medline

Knudsen EI (2004) Sensitive periods in the development of the brain and behavior. J Cogn Neurosci 16:1412-1425. CrossRef Medline

Kuhl PK (2010) Brain mechanisms in early language acquisition. Neuron 67:713-727. CrossRef Medline

Kurth S, Ringli M, Geiger A, LeBourgeois M, Jenni OG, Huber R (2010) Mapping of cortical activity in the first two decades of life: a high-density sleep electroencephalogram study. J Neurosci 30:13211-13219. CrossRef Medline

Kurth S, Ringli M, Lebourgeois MK, Geiger A, Buchmann A, Jenni OG, Huber R (2012) Mapping the electrophysiological marker of sleep depth reveals skill maturation in children and adolescents. Neuroimage 63:959965. CrossRef Medline

Landsness EC, Crupi D, Hulse BK, Peterson MJ, Huber R, Ansari H, Coen M, Cirelli C, Benca RM, Ghilardi MF, Tononi G (2009) Sleep-dependent improvement in visuomotor learning: a causal role for slow waves. Sleep 32:1273-1284. Medline

Määttä S, Landsness E, Sarasso S, Ferrarelli F, Ferreri F, Ghilardi MF, Tononi 
G (2010) The effects of morning training on night sleep: a behavioral and EEG study. Brain Res Bull 82:118-123. CrossRef Medline

Maret S, Faraguna U, Nelson AB, Cirelli C, Tononi G (2011) Sleep and waking modulate spine turnover in the adolescent mouse cortex. Nat Neurosci 14:1418-1420. CrossRef Medline

Marshall L, Helgadóttir H, Mölle M, Born J (2006) Boosting slow oscillations during sleep potentiates memory. Nature 444:610-613. CrossRef Medline

Mascetti L, Muto V, Matarazzo L, Foret A, Ziegler E, Albouy G, Sterpenich V, Schmidt C, Degueldre C, Leclercq Y, Phillips C, Luxen A, Vandewalle G, Vogels R, Maquet P, Balteau E (2013) The impact of visual perceptual learning on sleep and local slow-wave initiation. J Neurosci 33:33233331. CrossRef Medline

Miyamoto H, Katagiri H, Hensch T (2003) Experience-dependent slowwave sleep development. Nat Neurosci 6:553-554. CrossRef Medline

Nere A, Hashmi A, Cirelli C, Tononi G (2013) Sleep-dependent synaptic down-selection (I): modeling the benefits of sleep on memory consolidation and integration. Front Neurol 4:143. CrossRef Medline

Ngo HV, Martinetz T, Born J, Mölle M (2013) Auditory closed-loop stimulation of the sleep slow oscillation enhances memory. Neuron 78:545553. CrossRef Medline

Nicholas JG, Geers AE (2007) Will they catch up? The role of age at cochlear implantation in the spoken language development of children with severe to profound hearing loss. J Speech Lang Hear Res 50:1048-1062. CrossRef Medline

Penhune VB (2011) Sensitive periods in human development: evidence from musical training. Cortex 47:1126-1137. CrossRef Medline

Rakic P, Bourgeois JP, Goldman-Rakic PS (1994) Synaptic development of the cerebral cortex: implications for learning, memory, and mental illness. Prog Brain Res 102:227-243. CrossRef Medline

Rasch B, Born J (2013) About sleep's role in memory. Physiol Rev 93:681766. CrossRef Medline
Schlaug G, Jäncke L, Huang Y, Staiger JF, Steinmetz H (1995) Increased corpus callosum size in musicians. Neuropsychologia 33:1047-1055. CrossRef Medline

Schmidt C, Peigneux P, Muto V, Schenkel M, Knoblauch V, Münch M, de Quervain DJ, Wirz-Justice A, Cajochen C (2006) Encoding difficulty promotes postlearning changes in sleep spindle activity during napping. J Neurosci 26:8976-8982. CrossRef Medline

Sowell ER, Trauner DA, Gamst A, Jernigan TL (2002) Development of cortical and subcortical brain structures in childhood and adolescence: a structural MRI study. Dev Med Child Neurol 44:4-16. CrossRef Medline

Steele CJ, Bailey JA, Zatorre RJ, Penhune VB (2013) Early musical training and white-matter plasticity in the corpus callosum: evidence for a sensitive period. J Neurosci 33:1282-1290. CrossRef Medline

Steriade M, Nuñez A, Amzica F (1993) A novel slow ( $<1 \mathrm{~Hz})$ oscillation of neocortical neurons in vivo: depolarizing and hyperpolarizing components. J Neurosci 13:3252-3265. Medline

Taylor JA, Ivry RB (2012) The role of strategies in motor learning. Ann N Y Acad Sci 1251:1-12. CrossRef Medline

Taylor JA, Krakauer JW, Ivry RB (2014) Explicit and implicit contributions to learning in a sensorimotor adaptation task. J Neurosci 34:3023-3032. CrossRef Medline

Thomas KM, Hunt RH, Vizueta N, Sommer T, Durston S, Yang Y, Worden MS (2004) Evidence of developmental differences in implicit sequence learning: an fMRI study of children and adults. J Cogn Neurosci 16:1339_ 1351. CrossRef Medline

Vyazovskiy VV, Olcese U, Lazimy YM, Faraguna U, Esser SK, Williams JC, Cirelli C, Tononi G (2009) Cortical firing and sleep homeostasis. Neuron 63:865-878. CrossRef Medline

Wilhelm I, Diekelmann S, Born J (2008) Sleep in children improves memory performance on declarative but not procedural tasks. Learn Mem 15:373-377. CrossRef Medline 